\title{
Relative Contributions of Coupled Rotations and Small-Amplitude Torsions to Conformational Relaxation in Polymers
}

\author{
Turkan Haliloglu, Ivet Bahar, and Burak Erman
}

Polymer Research Center and School of Engineering, Bogazici University, and TUBITAK Advanced Polymeric Materials Research Center, Bebek 80815, I stanbul, Turkey

Wayne L. Mattice*

Institute of Polymer Science, The University of Akron, Akron, Ohio 44325-3909

Received J uly 12, 1996; Revised Manuscript Received October 21, $1996^{\otimes}$

\begin{abstract}
Local dynamics of polyethylene and 1,4-trans-polybutadiene chains are analyzed by the cooperative kinematics approach. The method includes the effects of environmental friction and the conformational energy changes involved in bond rotational motions. A given bond is rotated by $120^{\circ}$ and the response of the surrounding bonds along the chain, which are necessary for localizing the motion, are analyzed. The conformational responses are classified into two sets, one exhibiting a coupled rotation of another bond, i.e involving a rotational barrier crossing, and the other accommodated by collective small-amplitudetorsions (librational) of several bonds in the neighborhood of the rotating bond. Coupled rotations are shown to increase significantly with increasing environmental frictional resistance. Conversely, librational motions are dominant for localization in less viscous environments. An important finding is that, for polyethylene, the subset of transitions in which the relaxation is achieved by torsional librations only has the same mechanism as that of the complete set of transitions. Thus, cooperative torsional librations may localize a motion as efficiently as coupled rotational jumps in polyethylene, al though their proportion may be low in highly viscous environments. This is in support of the postulate advanced recently by Moe and Ediger [Mœ, N. E.; Ediger, M. D. Macromolecules 1996, 29, 5484] on local dynamics of polyisoprene, in the sense that libration alone can dissipate local disturbances in the conformations. However, for 1,4-trans-polybutadiene, the set of motions including librations only exhibit some departure from that of the overall set of transitions. The size of the correlated sequence of bonds responding to the isomerization of the central bond by libration is about eight bonds and is symmetrically distributed on both sides of the central bond for polyethylene and slightly biased for one side in poly 1,4-trans-polybutadiene. Coupled barrier crossing rotations, on the other hand, take place either on one side of the central bond only in the former or absolutely in one side in the latter and hence involve a shorter correlation length al ong the chain.
\end{abstract}

\section{Introduction}

The effects of rotameric transitions in polymer chains are highly localized. The crankshaft model $\mathrm{s}^{1,2}$ and the more elaborate models of Helfand, ${ }^{3}$ which included rotameric transitions and librations, were early studies inviting attention to the importance of constraints imposed by chain connectivity and frictional effects in determining the mechanism of conformational motions. Subsequent advances in computations have led to a more precise understanding of local dynamics. ${ }^{4,5}$ It is now firmly established that the bond torsional degrees of freedom are mostly responsible for the observed relaxation phenomena; bond rotameric transitions from one isomeric state to another are confined, or localized, to within about ten bonds along the backbone, and the tails on each side of this mobile segment are not appreciably affected.

The observed relaxation phenomena result from the interplay between two major effects of different origin: ${ }^{6}$ The first is the environmental frictional resistance favoring motions which minimize the atomic displacements, in general. This is an effect of intermolecular origin. The second, intramolecular, is the conformational energy change involved in bond rotational motions. In this case, certain isomeric states and transitions are preferred due to the particular geometry and chemical nature of the chain, irrespective of the fric-

\footnotetext{
${ }^{\otimes}$ Abstract published in Advance ACS Abstracts, December 1, 1996.
}

tional constraints. Clearly, the former effect dominates the mechanism of motion in the bulk, whereas the second operates, alone, in the other extreme case of a chain in vacuum.

Two mechanisms have been advanced for accomplishing the localization of the motion succeeding an isomeric jump: The first involves the cooperative transition of a neighboring bond along the sequence from one isomeric state to another. These are shortly referred to as coupled rotations. In the second group, the localization is achieved by the collective small amplitude motion of several bonds surrounding the rotating bond. These will be referred to as small-amplitudetorsions or librational motions. They do not involve a crossing of a rotational energy barrier, but simply the changes in dihedral angles within the potential energy wells of the bond rotational isomeric states.

In a recent study, the relative role of coupled rotations, on the one hand, and cooperative small-amplitude torsions, on the other, has been explored. ${ }^{7}$ Molecular dynamics (MD) trajectories have been analyzed with filtering techniques so as to extract the contribution of different modes of motion on the time evolution of macromolecular conformations. One major observation is that the small-amplitude torsions are not random. Instead, they are highly directed so as to accommodate the conformational changes induced by isomeric jumps with a minimum distortion of the overall configuration. As a consequence, the bond orientations are somewhat preserved on a local scale, and the correlations associ- 
ated with their reorientations, including those of $\mathrm{C}-\mathrm{H}$ bonds, persist for a longer duration. The corresponding orientational correlation (OC) times, $\tau_{\mathrm{c}}$, are clearly longer than those that would be observed in the absence of such cooperative small-amplitude torsions. Interestingly, the effect of the same small-amplitude torsions on the time decay of conformational correlations (CC) is of opposite character. Here, conformational correlations refer to those existing between the time-delayed rotameric states, trans, gauche ${ }^{ \pm}$, etc. of individual bonds. The interference of small-amplitude torsions leads, in this case, to a faster decay of correlations; these are obviously manifested by shorter CC times. These observations reveal that the time dependence of the two types of correlation functions, OC and CC, exhibit different trends, even though the same relaxation mechanism, characteristic of a given chain geometry and energetics in a given environment, takes place.

In a recent study, Moe and Ediger approached the same issue, i.e the role of rotameric transitions in local chain dynamics, as opposed to that of coupled librations, from a different perspective. ${ }^{8}$ Based on the observation that CC times in vacuum, solution, and melt for polyisoprene do not correlate with the OC times obtained in the same media, Moe and E diger questioned whether conformational transitions were mandatory for the reorientation of $\mathrm{C}-\mathrm{H}$ bonds. However, thefact that $\mathrm{CCS}$ and OCs do not obey the same time dependence does not necessarily indicate the absence of rotational jumps. In fact, it has been demonstrated ${ }^{7}$ that the two correlation functions are differently affected by small-amplitude torsions.

A quantitative test that is pertinent to the relative importance of these two types of relaxation mechanism in local chain dynamics is possible by the use of the cooperative kinematics approach.6,9-11 The latter provides precise information on the mechanism of relaxation at the atomic scale and is in good agreement with results from MD simulations and experiments for the types of local conformational changes of interest here ${ }^{11-13}$ It reproduces ${ }^{11}$ the cooperativity of the local torsions seen in a molecular dynamics simulation of 1,4-cispolybutadiene at bulk density. ${ }^{12}$ This is an analytical approach based on energy minimization and yields a unique solution, the most probable conformational rearrangement of a chain in response to a local perturbation, for a known structure in a medium characterized by an effective friction coefficient. That approach is used here to determine whether the relaxation of a chain following a rotameric transition at bond i does, or does not, involve a rotameric transition at another bond.

The model and method are briefly outlined in the next section followed by the results for two types of chains, polyethylene (PE) and poly 1,4-trans-polybutadiene (trans-PB), illustrating the distinct behavior of different structures. The mechanism of motion will be analyzed on the basis of two properties observed in the absence and in the presence of small-amplitude torsions: (i) the optimal distribution of dihedral angle changes along the chain in response to a rotational isomeric jump, and (ii) the most probable mean-square displacements of backbone atoms accompanying a rotameric transition. It will be shown that the subset of conformational motions which do not involve a rotational energy barrier crossing may exhibit the qualitative features of the relaxation mechanism to some extent. Yet, the precise mechanism of relaxation, characteristic of a given structure, will be

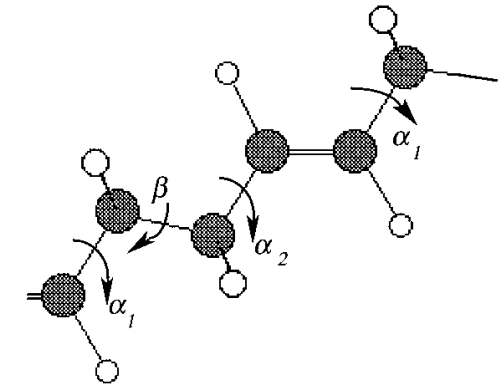

Figure 1. A segment of trans-PB chain. Three types of backbone bonds exist in each repeat unit: double bond connecting two $\mathrm{sp}^{2}$ carbon atoms, $\beta$ bond connecting two $\mathrm{sp}^{3}$ carbon atoms, and $\alpha_{1}$ and $\alpha_{2}$ bonds connecting $\mathrm{sp}^{2}$ and $\mathrm{sp}^{3}$ carbon atoms.

shown to involve a large number of coupled rotations, particularly in highly viscous environment.

\section{Model and Method}

Chain Models. Two model chains are considered: $P E$, chosen for the symmetry and simplicity of the backbone geometry, and $\mathrm{PB}$, reflecting the effects of the different geometry and torsional energies. The geometric variables are defined in the usual manner, with I, $\theta$, and $\phi$ denoting the backbone bond length, bond angle, and torsion angle, respectively. A segment of the transPB is presented in Figure 1. The bonds labeled $\alpha_{1}$ and $\alpha_{2}$ in the figure are structurally equival ent; these are referred to as $\alpha$ bonds. The $\mathrm{CH}_{2}-\mathrm{CH}_{2}$ bond between two a bonds is referred to as a $\beta$ bond. A repeat unit consists of one double bond, one bond of type $\beta$, and two bonds of type $\alpha$. Bond rotational potentials are expressed by a fifth-order polynomial as

$$
\mathrm{V}\left(\phi_{\mathrm{i}}\right)=\mathrm{k}_{\phi} \sum_{\mathrm{m}=0}^{5} \mathrm{a}_{\mathrm{m}} \cos ^{\mathrm{m}} \phi_{\mathrm{i}}
$$

The energy and geometry parameters for PE and PB are given in our previous work. ${ }^{6,11}$

Theory and Method. The theory gives the most probable changes in the dihedral angles of a chain of $n$ bonds in a given configuration when the ith bond undergoes a rotation by an increment of $\delta \phi_{\mathrm{i}}$. The changes are subject to the condition that the work $\delta \mathrm{W}$ done against friction plus the change $\delta \mathrm{V}$ in the conformational energy $\mathrm{V}=\sum_{\mathrm{i}} \mathrm{V}\left(\phi_{\mathrm{i}}\right)$ is a minimum. This condition is expressed as

$$
\frac{\partial \mathrm{V}}{\partial \phi_{\mathrm{i}}}+\frac{1}{2} \frac{\partial \delta \mathrm{W}}{\partial \phi_{\mathrm{i}}}=0 \quad 2 \leq \mathrm{i} \leq(\mathrm{n}-1)
$$

and

$$
\frac{\partial \delta W}{\partial \delta \Phi}=\frac{\partial \delta W}{\partial \delta \Psi}=\frac{\partial \delta W}{\partial \delta \chi}=\frac{\partial \delta W}{\partial \delta \mathbf{R}_{0}}
$$

Here $\delta \mathrm{W}$ denotes the differential work done by the chain against friction during the small displacements $\delta \mathbf{R}_{\mathrm{i}}, 1$ $\leq \mathrm{i} \leq \mathrm{n}$, accompanying the rotation $\delta \phi_{\mathrm{i}} . \Phi, \Psi$, and $\chi$ are the Euler angles defining the absolute orientation of the chain with respect to a laboratory-fixed frame. The last equality in eq 3 refers to the three derivatives of $\delta \mathrm{W}$ with respect to the components of the absolute position vector $\mathbf{R}_{0}$. $\delta \mathrm{W}$ is expressed in terms of the 
effective friction coefficient $\xi$ as

$$
\delta \mathrm{W}=(\xi / \delta \mathrm{t}) \sum_{\mathrm{i}} \delta \mathbf{R}_{\mathrm{i}} \cdot \delta \mathbf{R}_{\mathrm{i}}
$$

where $\delta \mathrm{t}$ is the time interval associated with $\delta \phi_{\mathrm{i}}$. Equation 2 is conveniently rewritten as

$$
\mathrm{k}_{0} \frac{\delta \mathrm{V}}{\partial \phi_{\mathrm{i}}}+\frac{\partial \delta \mathrm{W}}{\partial \phi_{\mathrm{i}}}=0 \quad 2 \leq \mathrm{i} \leq(\mathrm{n}-1)
$$

where the dimensionless ratio

$$
\mathrm{k}_{0}=\frac{2 \mathrm{k}_{\phi}}{\xi / \delta \mathrm{t}}
$$

gives a measure of the relative strengths of conformational and frictional effects. The solution of eqs 3 and 5 gives the complete set of variables $\left\{\phi_{2}, \phi_{3}, \ldots, \phi_{\mathrm{n}-1}, \Phi\right.$, $\left.\Psi, \chi, \mathbf{R}_{0}\right\}$ characterizing the optimal configuration assumed by the chain in response to the torsion $\delta \phi_{\mathrm{i}}$. For a complete derivation of the above equations and a detailed presentation of the mathematical formal ism of the CK approach, the reader is referred to our previous work.6,9

Calculations are performed by applying successive rotations $\delta \phi_{\mathrm{i}}$ of $3^{\circ}$ until the completion of a rotameric transition of $120^{\circ}$ and solving eq 1 at each step. Chains of $n=25$ bonds are conveniently considered, the motion being localized to a segment of \pm 5 bonds surrounding the rotating (ith) bond. Previous CK calculations have demonstrated that the mechanism of motion is not affected by the overall size of the molecule. Calculations are repeated for a set of 1000 representative configurations so as to extract the average behavior of the chain, irrespective of the original conformation. In principle, a set of 200 different configurations is sufficient to yield a reasonable estimate of the most probable mechanism of motion; considering a larger number of configurations increases the accuracy. Transitions violating the volume exclusion requirements are rejected. The acceptable transitions are classified into two subsets. Subset (A) includes those transitions accompanied by the rotameric jump of at least one other bond along the chain, and (B) those accommodated by small-amplitude torsions or librations without invoking the crossing of a rotational energy barrier. Results obtained for the subsets $(A)$ and $(B)$ will be compared in the following.

\section{Results}

General Classification of Conformational Transitions. The fractions $x(A)$ and $x(B)$ of transitions lying in the respective subsets $(A)$ and $(B)$ depend on the effective friction coefficient and on the specific chain structure and energetics. Results obtained for PE and trans-PB in different viscous environments are presented in Figure 2 with heavy and light solid lines, respectively. Here, the ratios $x(B) / x(A)$ are shown as a function of the effective friction coefficient. The abscissa represents the reciprocal of the dimensionless parameter $k_{0}$ defined by eq 6 . It is expressed in logarithmic coordinates inasmuch as it spans several orders of magnitude. $\mathrm{k}_{0}{ }^{-1}$ approaches zero in vacuum $(\xi=0)$, as well as in the presence of infinitely high rotational barriers. In this limiting case, $x(B)$ approaches unity; i.e, no local ization phenomenon is operative and rotational jumps may be accommodated by the accompanying small-amplitude torsions of the surrounding bonds along the chain. In the opposite extreme case of very

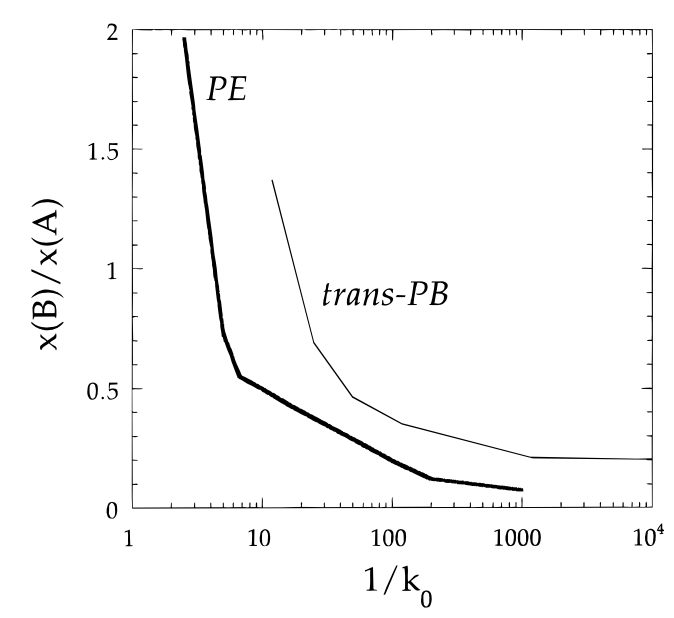

Figure 2. The fractions of $x(B)$ and $x(A)$ as a function of the effective friction coefficient for PE (heavy solid line) and for trans-PB (light solid line).

low conformational barriers in a highly dense environment, $\mathrm{k}_{0}{ }^{-1}$ approaches infinity and conformational transitions occur necessarily via coupled rotations of two or more bonds so as to achieve the most efficient Iocalization. In this limit $x(A)$ approaches unity and the ratio $x(B) / x(A)$ approaches zero. At intermediate friction coefficients and rotational energy barriers, the proportion of rotameric jumps increases with increasing friction coefficient (or decreasing $k_{0}$, corresponding to an increase of environmental friction relative to barrier heights).

The two curves in Figure 2 separate into three regimes: (i) the low friction regime where rotational jumps are easily accommodated by bond torsional motions without involving a barrier crossing, (ii) the intermediate regime where both effects of friction and barrier heights are operating with a dominance of rotational jumps, and (iii) the high-friction regime where coupled transitions are most pronounced. The ratio $x(B) / x(A)$ equates to unity, i.e. the two processes of relaxation are equally probable, for $\mathrm{k}_{0}$ values falling into the low-friction regime for both chains. We note that the range $10 \leq 1 / k_{0} \leq 100$ approximates the bulk PE density and frictional conditions, and is shifted to lower values, by at least one order of magnitude, in solution. ${ }^{6}$ For trans-PB, $1 / k_{0}$ values representative of the bulk state lie in the range $10 \leq 1 / \mathrm{k}_{0} \leq 10^{3}$, approximately, which in parallel with PE, falls in the intermediate density regime displayed in the figure.

Local Dynamics of PE. Figure 3 displays the changes in bond dihedral angles triggered by the rotameric transition of a given $(i=13)$ bond in a PE chain, using $1 / k_{0}=100$. The solid line with open circles represents the behavior averaged over all $10^{3}$ original configurations, irrespective of the mechanism (A) or (B) of the transition. The ordinate represents the cumulative changes $\Delta \phi_{\mathrm{j}}, \mathrm{j} \neq \mathrm{i}$, in the dihedral angles after completion of the full rotameric transition of bond $\mathrm{i}$. The line with triangles refers to the subset $(B)$ of transitions which do not involve a rotational energy barrier crossing by any bond in the neighborhood of the rotating bond. Here only those transitions exhibiting a cumulative dihedral angle change of $\Delta \phi_{\mathrm{j}}<\Delta \phi_{\mathrm{c}}=60^{\circ}$ are included. The limiting value of $\Delta \phi_{c}$ is the angular position of the saddle point on the energy surface between isomeric states. A more stringent test, $\Delta \phi_{\mathrm{j}}<45^{\circ}$ for example, yiel ds the dashed curve with filled circles, which reproduces the same qualitative behavior. Mainly, the 


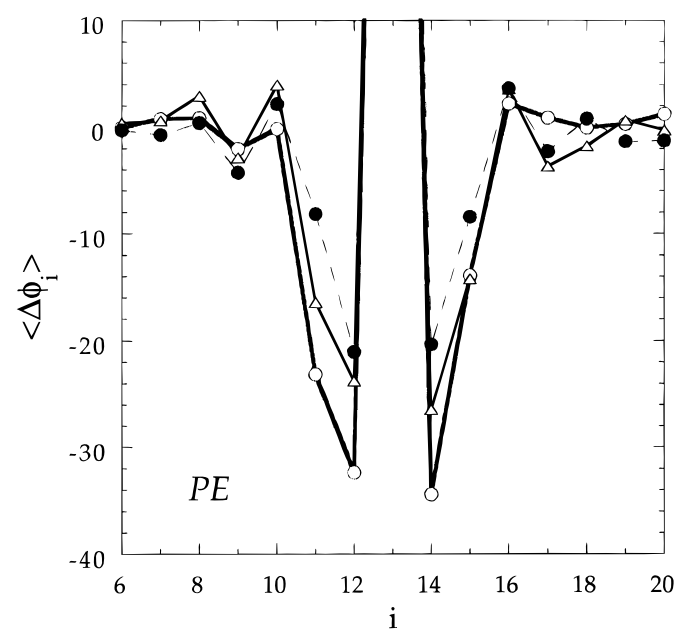

Figure 3. Average changes in dihedral angles, $\left\langle\Delta \phi_{\mathrm{i}}\right\rangle$, in response to $120^{\circ}$ rotation of a given bond $(i=13)$ in $P E$ with $1 / k_{0}=100$. The solid line with open circles represents the behavior irrespective of the mechanism (A) or (B) of the transitions. The line with triangles and the dashed curve with filled circles refer to the subset $(B)$ of transitions which do not involve a rotational energy barrier crossing by any bond in the neighborhood of the rotating bond and exhibiting a cumulative dihedral angle change $\Delta \phi_{\mathrm{j}}<60^{\circ}$ and $\Delta \phi_{\mathrm{j}}<45^{\circ}$, respectively

subsets of transitions in which the relaxation is achieved by torsional librations only exhibit the same mechanism, on the average, as the complete set of transitions. The first two neighboring bonds on either side of bond i undergo counterrotations, whose amplitudes are slightly reduced by precluding coupled rotameric jumps.

Thus, cooperative torsional librations are shown to be able to localize a motion as efficiently as coupled rotational jumps in PE, although their proportion may be relatively low in highly viscous environments. The qualitative behavior illustrated in Figure 3 is verified to be valid for a broad range of effective friction coefficients, the effect of the environmental resistance being in the form of varying the proportion of transitions belonging to subsets $(A)$ and $(B)$ rather than altering the mechanism of relaxation. The latter appears to be a property determined by the specific geometry and conformational energetics of the examined chain. We note that the behavior of the subset $(A)$ is not explicitly shown in Figure 3 , the latter being almost indistinguishable from that of the overall set displayed by the solid line with open circles when $\mathrm{k}_{0}^{-1}=100$.

In addition to the changes in dihedral angles, the spatial displacements of chain units in the neighborhood of a rotating bond provide information on the mechanism of motion. Figure 4 displays the mean-square atomic displacements obtained for the three cases described in Figure 3 as a function of atom indices. Again the solid line with open circles represents the overall behavior, while the upper two curves refer to the response of the chain through small-amplitude torsions only. Clearly, the atoms 13 and 14 at both ends of the bond undergoing the rotameric transition undergo the largest translation $(\sim 1 \AA)$ in space; and the atomic displacements are smaller than $0.2 \AA$ beyond sixth neighbors on both sides. A distinct feature appearing in this subset (B) of transitions in comparison to the overall set is the peak at the atomic positions 11 and 16. These peaks become more pronounced as smaller torsional librations, only, are permitted. The solid line for the overall set demonstrates that the displacement of this pair of atoms is efficiently minimized provided

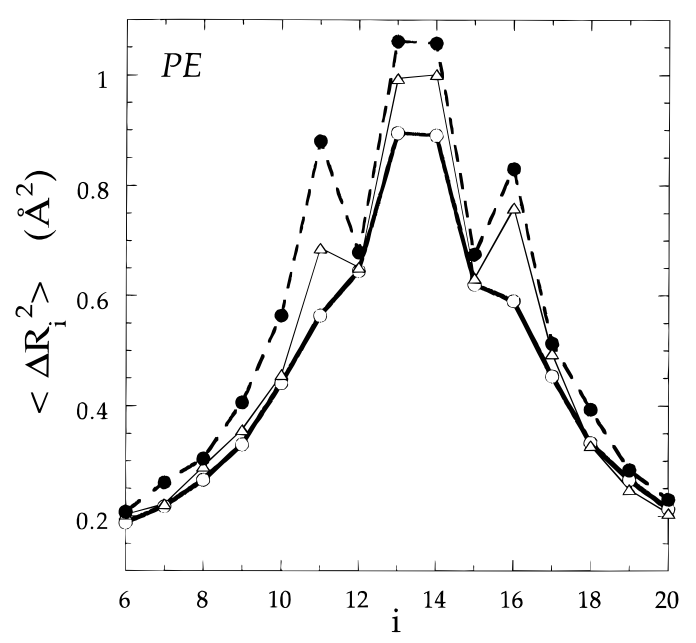

Figure 4. Average spatial displacements of atoms in response to the rotation of a given bond $(i=13)$ in PE with $1 / k_{0}=100$ for the three cases described in Figure 3.

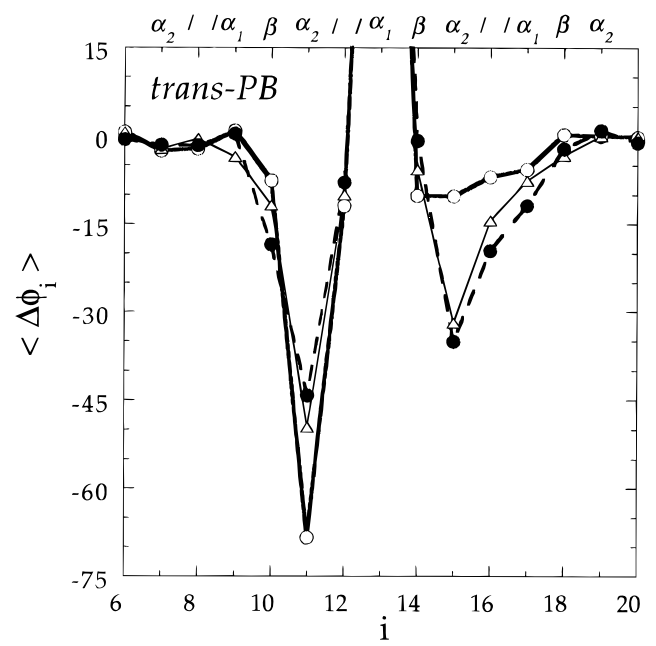

Figure 5. Average changes in the dihedral angles of bonds, $\left\langle\Delta \phi_{i}\right\rangle$, in response to a $120^{\circ}$ rotation of the $\alpha_{1}$ bond in trans-PB with $1 / k_{0}=1190$ for the cases described in Figure 3 for PE.

that coupled rotations are operative. Thus, although the general characteristics of the subsets $(A)$ and $(B)$ are comparable insofar as the size of mean-square displacements and their decrease by increasing separation from the rotating bond are concerned, the detailed distribution of atomic displacements is affected by the precise mechanism of relaxation.

Calculations were repeated for various $1 / k_{0}$ values, which verified that the same conclusions are valid for different viscous environments, except for a uniform change in the amplitudes of the motions depending on the effective friction coefficient. As shown in previous studies, the net effect of an increase in frictional resistance is a decrease in atomic displacements and an enhancement in coupled rotations, leading to larger amplitude dihedral angle changes on the average. However, the detailed shapes of the curves obtained for the two subsets of transitions (A) and (B) remain unaffected. Filtering procedures have also been employed by Ediger for the analysis of the local internal dynamics of chains in various media. 5,14

Local Dynamics of trans-PB. Cumulative changes in the dihedral angles after completion of the full rotameric transition of an $\alpha_{1}$ bond of a PB chain is presented in Figure 5 in the same format as that of Figure 3 , for $1 / k_{0}=1190$. The types of backbone bonds 


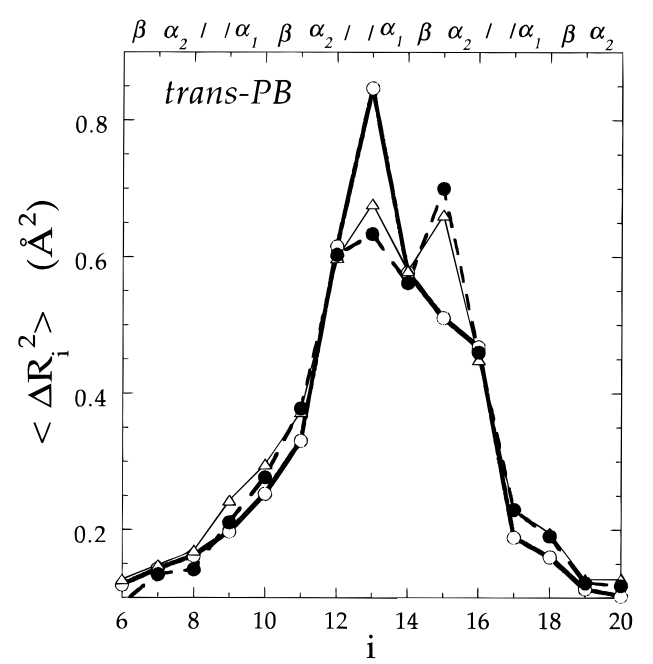

Figure 6. Average spatial displacements of atoms in response to the rotation of $\alpha_{1}$ bonds in trans-PB are displayed in the same format as that of Figures 3-5.

are shown in the upper abscissa scale for clarity. The solid line with open circles exhibits a strong asymmetry, resulting from the specific geometry and energy characteristics of the trans-PB repeat unit. The $\alpha_{1}$ bond that is rotated is indexed as $i=13$ along the abscissa. The largest response is observed in the bond $(i-2)$ corresponding to the nearest $\alpha_{2}$ bond across the double bond. A weak response of the $\alpha_{2}$ bond across the $\beta$ bond, located at $(i+2)$, is also observable. The rotations of both bonds $(i \pm 2$ ) are in the opposite direction to that of the rotating ith bond. The response of the $\beta$ bond is negligibly small due to the higher energy barrier required for a rotameric transition and due to the specific geometry of the PB chain. 6,11 The line through the triangles in the figure represents the results obtained for the subset $(B)$. According to this curve, the amplitude of the counterrotation of the $\alpha_{2}$ bond across the double bond is diminished and that accross the $\beta$ bond is strongly accentuated. The dashed curve obtained for rotations less than $45^{\circ}$ exhibits the same features as that of subset (B).

It is interesting to note that the dihedral angle changes on both sides of the rotating bond are of comparable magnitude for the conformational motions bel onging to subset (B). The curves obtained for subset (A) are almost indistinguishable from the solid curve displayed for the overall ensemble. Thus, the asymmetric response of the chain is induced by coupled bond rotations and cannot be interpreted in terms of small amplitude torsions alone.

In Figure 6, the mean-square atomic displacements accompanying the rotation of the 13th bond connecting backbone carbons are presented as a function of atom indices. Due to the asymmetry of the chain, the atoms 13 and 14 at the ends of the rotating bond exhibit different displacements. The three curves refer to the three cases described in Figure 5. The solid line with open circles shows the average behavior of all chains, whereas the other two lines reflect the behavior of the subset of transitions which do not involve another barrier crossing. Similarly to Figure 4, a new peak emerges in the absence of simultaneous rotameric jumps, at the 15th carbon atom. This is the $\mathrm{CH}_{2}$ unit located between the bonds $\beta$ and $\alpha_{2}$ contiguous to the rotating bond $\alpha_{1}$. The displacement of this particular atom is not efficiently minimized unless conformational motions involving the crossing of at least one other rotational energy barrier are operative.

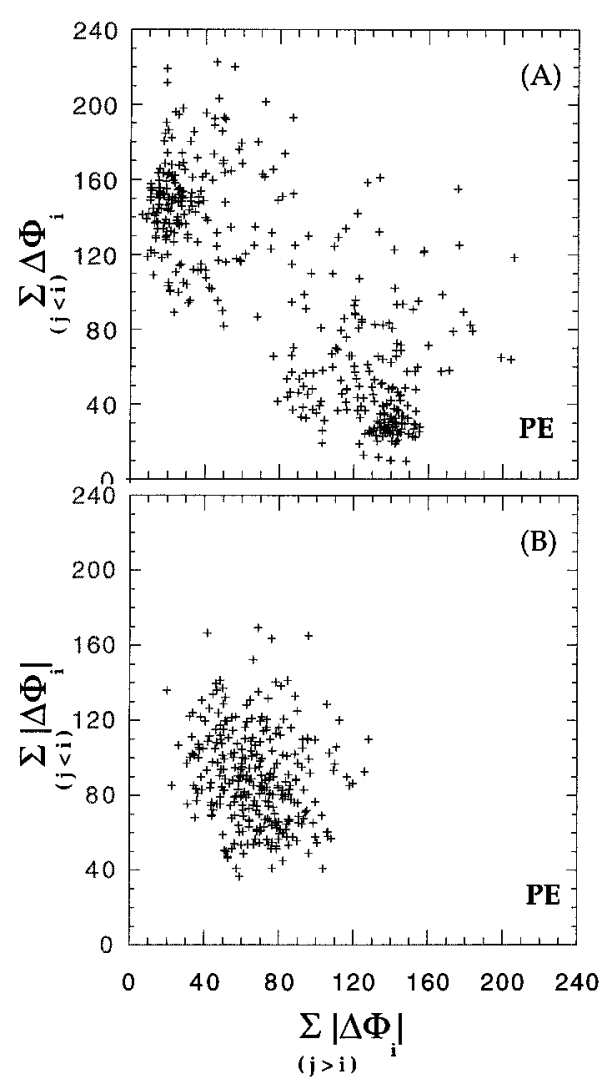

Figure 7. The overall response of all bonds $j$ located on the left $(\mathrm{j}<\mathrm{i})$, or right $(\mathrm{j}>\mathrm{i})$, of the rotating bond for PE using $\mathrm{k}_{0}{ }^{-1}=5$ is represented in two panels: (A) for the subset of motions which involve a barrier crossing, $\left|\Delta \phi_{\mathrm{j}}\right| \leq 60$ for at least one bond $\mathrm{i} \neq \mathrm{j},(\mathrm{B})$ the transitions accommodated by torsional librations, only, $\left|\Delta \phi_{\mathrm{j}}\right| \leq 60$ for all $\mathrm{j} \neq \mathrm{i}$.

Global View of the Two Relaxation Mechanisms. Effective Correlation Lengths. Figures 3-6 suggest that the isomeric transition of a given bond is accompanied by the coupled rotation, with or without barrier crossing, of \pm 2 neighboring bonds along the backbone. The atomic displacements, on the other hand, are confined to about \pm 4 neighbors in PE, and \pm 3 neighbors in trans- $P B$, if we neglect the displacements $\left\langle\Delta \mathbf{R}_{\mathrm{j}}{ }^{2}\right\rangle^{1 / 2} \leq 0.4 \AA$. Although these figures provide an estimate of the size of the chain segment, or the correlation length, efficiently accommodating a rotameric jump, a more detailed analysis will be undertaken here, which will elucidate a fundamental difference in the character of the two types $(A)$ and $(B)$ of conformational relaxation.

We now focus on the overall response of all bonds $j$ located on the left $(j<i)$, or right $(j>i)$, of the rotating bond. In order to acquire an estimate of the size of dihedral angle changes, irrespective of the sense of rotation, results are consol idated into the forms $\sum_{\mathrm{j}}\left|\Delta \phi_{\mathrm{j}}\right|$ for the two sides $(j<i)$ and $(j>i)$. These will be shortly referred to as $\Delta \Phi(j<i)$ and $\Delta \Phi(j>i)$. Calculations performed for $P E$ using $k_{0}{ }^{-1}=5$ lead to Figure 7. This value of $\mathrm{k}_{0}{ }^{-1}$ is shown in Figure 2 to yield nearly equal numbers of the types (A) and (B) of transitions. Here the abscissa and ordinate represent the cumulative changes $\sum_{\mathrm{j}}\left|\Delta \phi_{\mathrm{j}}\right|$ in the right and left portions of the chain, respectively, and the results obtained for different configurations are plotted in two panels. The upper part is obtained for the subset of motions $(A)$ which involve a barrier crossing, i.e. $\left|\Delta \phi_{\mathrm{j}}\right| \geq 60^{\circ}$, for at least one bond $\mathrm{j} \neq \mathrm{i}$. The lower panel refers to the the subset of 
transitions accommodated by torsional librations only. In this case, $\left|\Delta \phi_{j}\right|<60^{\circ}$ for all $\mathrm{j} \neq \mathrm{i}$.

A striking feature emerging in Figure 7 is that a bimodal distribution of cumulative dihedral angle changes is observed in subset (A), whereas subset (B) yields a unimodal distribution centered about $\Delta \Phi(\mathrm{j}<\mathrm{i})$ $=\Delta \Phi(\mathrm{i}>\mathrm{j})=80^{\circ}$, approximately. The two clusters in the upper panel indicate that the coupled rotations occur either on the left or on the right part of the rotating bond, with $\Delta \Phi(\mathrm{j}<\mathrm{i})$ and $\Delta \Phi(\mathrm{j}>\mathrm{i})$ values centered about $150^{\circ}$ and $25^{\circ}$, or vice versa. Thus, the apparent symmetry of the dihedral angle distributions in Figure 3 arises from the averaging of the response of both sides of the chain, although individual responses are actually confined to one side, left or right, of the rotating bond most likely, and the dihedral angle changes are about two times as large as those appearing in Figure 3. Small-amplitude torsions, on the other hand, exhibit a totally different behavior. The lower part of Figure 7, which exhibits a single cluster on the diagonal, indicates simply that this type of motion is evenly spread on both sides of the rotating bond, rather than concentrating on one side. The cumulative amplitude $\Delta \Phi(j<i)+$ $\Delta \Phi(j>i)$ is about $150^{\circ}$, in this case, which efficiently localize the structural perturbation induced by the isomeric jump of the bond i. An important corollary from these observations is that coupled rotations $(A)$ operate on a significantly shorter correlation length compared to librational motions, and therefore local ize the motion more efficiently. This is consistent with the preference for coupled motions in more viscous environments.

Figure 8 is the counterpart of Figure 7, obtained for trans-PB, with $1 / k_{0}=50$. The two types of relaxation mechanisms are of comparable importance at this $1 / \mathrm{k}_{0}$ value. In consistency with the distribution shown in Figure 5, the response of the chain is not symmetric with respect to the rotating bond (or diagonal in the figure), but is built on one side. This characteristic is even reflected on the librational motions, though significantly weakened.

\section{Discussion}

Cooperative kinematics analysis of configurational rearrangements following the rotameric transition of a central bond helps identify the different pathways of conformational relaxation. For isolated chains where the effect of friction from the environment is small compared to that of torsional barriers, the rotameric transition of a bond may be localized through small torsi onal arrangements of the surrounding bonds along the chain. At higher environmental friction, on the other hand, a rotameric jump is most probably accompanied by the coupled rotameric jump of a nearby bond along the chain. Yet, the cooperative torsional librations give the same mechanism as that of the complete set of transitions in PE, although their populations are low in the latter, and show slight deviations in trans-PB.

The number of bonds undergoing cooperative motion, either a jump or small-amplitude torsion, is in the range of 6-8 for both PE and trans-PB, in all environments. This observation is in agreement with results obtained

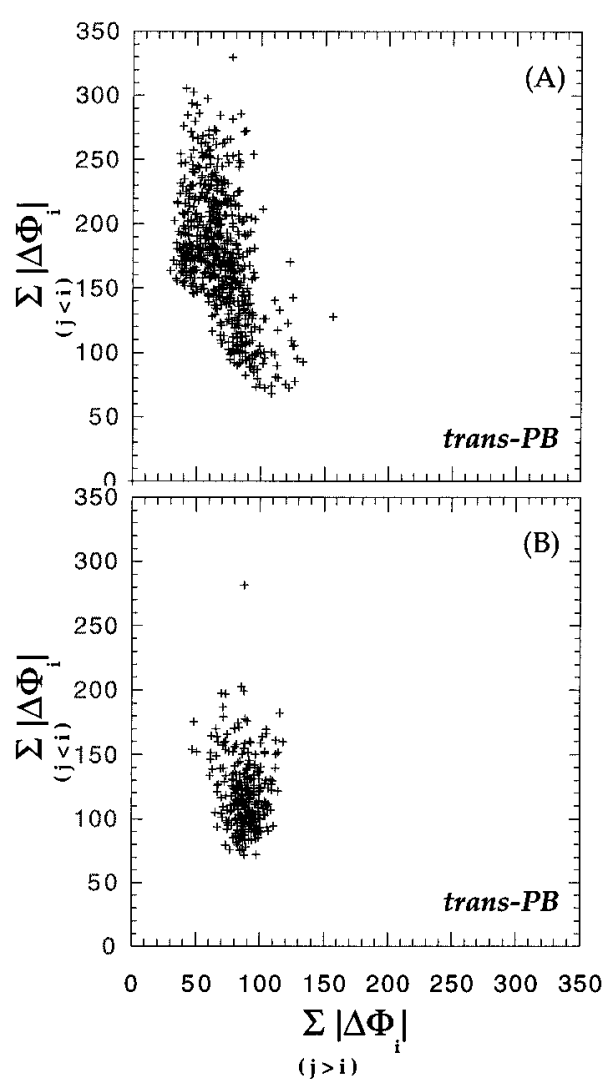

Figure 8. The overall response of all bonds $j$ located on the left $(j<i)$, or right $(j>i)$, of the rotating bond for trans-PB using $\mathrm{k}_{0}^{-1}=5$ is represented in two panels, $(A)$ and $(B)$, as described in the legend of Figure 7.

by Theodorou and Suter ${ }^{15}$ on simulations of deformed glassy polypropylene. A detailed analysis revealed different character of the two types (A) and (B) of conformational relaxation in both PE and trans-PB.

Acknowledgment. This work was supported by Grant INT 9312285 from the National Science F oundation.

\section{References and Notes}

(1) Boyer, R. F. Rubber Chem. Technol. 1963, 34, 1303.

(2) Schatzki, T. F. J . Polym. Sci. 1962, 57, 496.

(3) Helfand, E. J . Chem. Phys. 1971, 54, 4651.

(4) Bahar, I.; Erman, B.; Monnerie, L. Adv. Polym. Sci. 1994, $116,145$.

(5) Ediger, M. D.; Adolf, D. B. Adv. Polym. Sci. 1994, 116, 73.

(6) Bahar, I.; Baysal, N.; Erman, B.; Monnerie, L. Macromolecules 1995, 28, 1038.

(7) Baysal, C.; Atilgan, A. R.; Erman, B.; Bahar, I. Macromolecules 1996, 29, 2510.

(8) Moe, N. E.; Ediger, M. D. Macromolecules 1996, 29, 5484.

(9) Bahar, I.; Erman, B.; Monnerie, L. Macromolecules 1992, 25, 6309.

(10) Bahar, I.; Erman, B.; Monnerie, L. Macromol ecules 1992, 25, 6315.

(11) Baysal, C.; Bahar, I.; Erman, B.; Monnerie, L. Macromolecules 1996, 29, 2980.

(12) Kim, E.-G.; Mattice, W. L. J . Chem. Phys 1994, 101, 6242.

(13) Gee, R. H.; Boyd, R. H. J . Chem. Phys. 1994, 101, 8028.

(14) Adolf, D. B.; Ediger, M. D. Macromolecules 1992, 25, 1074.

(15) Theodorou, D. N.; Suter, U. W. Macromolecules 1986, 19, 379.

MA961021+ 